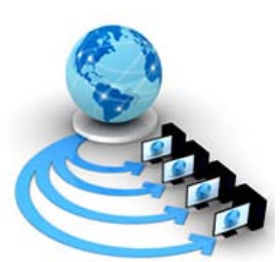

\title{
EFFICIENT TRACKING AREA MANAGEMENT FRAMEWORK FOR 5G NERWORK
}

\author{
K.RAVIKUMAR \\ Dept: Computer Science \\ Tamil University, \\ Thanjavur-613010 , India,
}

\author{
S.SARANYA \\ Dept: Computer Science \\ Tamil University, \\ Thanjavur-613010, India,
}

\begin{abstract}
To accommodate a various growing number of user equipment (UEs) on $5 g$ networks. Managing with the huge signaling overhead expected from UEs is a main problem to tackle hence as to achieve this objective. In this thesis, they develop aficeient tracking area list management (ETAM) system for 5 G cloud-based mobile networks. The proposed system contains of two parts. The first part is executed offline. The executed offline is responsible of assigning tracking areas (TAs) to TA lists (TALs). The second one is executed online. The executed online is responsible of the distribution of TALs on user equipment (UEs) during their movements across TA. For ffrest par t, they propose three keys, which are: (a) F-PAGING favoring the paging overhead over tracking area update (TAU), (b) F- TAU favoring TAU over paging, and (c) FOTA (i.e., Fair and Optimal Assignment of TALs to TAs) for a solution that uses bargaining game to ensure a fair tradeoff between TAU and paging overhead. For the second part, two solutions are proposed to assign in real time, TALs to different UEs. The computation load is kept lightweight in both solutions not to reduce the network performance. Also, both solutions do not need any additional new messages when assigning TALs to UEs. Thefirst solution takes into account only the priority between TALs. As for the second one, in additio n to the priority between TALs, it takes into account the UEs activities (i.e., in terms of incoming communication frequency and mobility patterns) to improve further the network performance. The presentation of ETAM is estimated through investigation and imitations, and the achieved results validate its feasibility and ability in achieving its thesis goals, improving the network performance by minimizing the cost related with paging and TAU.
\end{abstract}

KeyWords: Tracking area, Users Equipment, Mobile Network, Information Technology, Protocol, Frequency

\section{INTRODUCTION}

A set of connections is a collection of computers, servers, mainframes, network devices, peripherals, or other devices connected to one another to allow the sharing of data [1][3]. A network makes it possible to centralize data. All files shared by users are stored in a central location, which securities reliability and simplifies the update process [2]. Multiple levels of defense can be implemented on a network, making it more difficult to obtain unauthorized access to data [4]. A network can be operational with a backup system that runs at specific intervals, ensuring that critical data is available from a secondary source if needed [9].

Information technologies have become an integral part of our society, having a profound socio-economic impact, and elevating our daily lives with a plethora of services from media diversion (e.g. video) to more sensitive and safety-critical applications (e.g. e-commerce, e-Health, first responder services, etc.) [6]. If analysts' predictions are correct, just about each physical object they see (e.g. clothes, cars, trains, etc.) will also be related to the networks by the end of the decade (Internet of Things) [5]. Also, according to a Cisco forecast of the use of IP (Internet Protocol) networks by 2017, Internet traffic is evolving into a more dynamic traffic pattern [10]. The global IP traffic will communicate to 41 million DVDs per hour in 2017 and video communication will continue to be in the range of 80 to $90 \%$ of total IP traffic [8]. This market estimate will surely divide the growth in mobile traffic with current predictions suggesting a $1000 \mathrm{x}$ increase over the next decade [7].

\section{EXISTING SYSTEM}

The 4G system was originally envisioned by the Defense Advanced Research Projects Agency (DARPA). The DARPA selected the distributed architecture and end-to-end Internet protocol (IP), and believed at an early stage in peerto-peer networking in which every mobile device would be both a transceiver and a router for other devices in the network, eliminating the spoke-and-hub weakness of $2 \mathrm{G}$ and 3G cellular systems. Since the 2.5G GPRS system, cellular systems have provided dual infrastructures: packet switched nodes for data services, and circuit switched nodes for voice calls. In $4 \mathrm{G}$ systems, the circuit-switched infrastructure is abandoned and only a packet-switched network is provided, while 2.5G and 3G systems require both packet-switched and circuit-switched network nodes, i.e. two infrastructures in parallel. This means that in 4G, traditional voice calls are replaced by IP telephony.

\section{A) Demerits}

- Equipment has not been fully developed for network

- Network has more complex security issues

- Network protocols and standardization have not been defined

- $\quad$ Not many areas have 4 


\section{PROPOSED SYSTEM}

In this thesis, proposed an efficient tracking area list management (ETAM) system for $5 \mathrm{G}$ cloud-based mobile networks. The proposed system contains of two parts. The first part is executed offline. The executed offline is responsible of assigning tracking areas (TAs) to TA lists (TALs). The second one is executed online. The executed online is responsible of the distribution of TALs on user equipment (UEs) during their movements across TAs.For the first part, they pro pose three keys, which are: (a) FPAGING favoring the paging overhead over tracking area update (TAU), (b) F- TAU favoring TAU over paging, and (c) FOTA (i.e., Fair and Optimal Assignment of TALs to TAs) for a solution that uses bargaining game to ensure a fair tradeoff between TAU and paging overhead. For the second part, two solutions are proposed to assign in real time, TALs to different UEs. The computation load is kept lightweight in both solutions not to reduce the network performance. Also, both solutions do not need any additional new messages when assigning TALs to UEs. The first solution takes into account only the priority between TALs. As for the second one, in addition to the priority between TALs, it takes into account the UEs activities (i.e., in terms of incoming communication frequency and mobility patterns) to improve further the network performance.

\section{A) Merits}

- High efficiency

- Feasibility

- Network has complex security.

- Network protocols and standardization have been clear

- Many areas have 4G service

- Low cost

\section{EXPERIMENTAL RESULT}

\section{A) High Performance}

In these experiments cost. OTCM gives a result with throughput of 25\%.TCC gives a result with throughput of $40 \%$.MTC gives a result with throughput of 50\%.ETAM gives a result with throughput of $70 \%$.

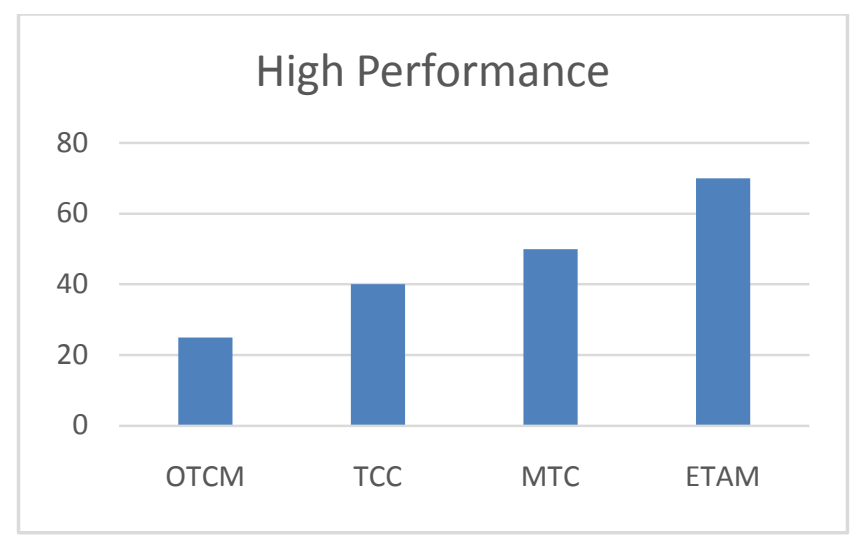

\section{B) Cost}

In these experiments cost. OTCM gives a result with throughput of 50\%.TCC gives a result with throughput of $60 \%$.MTC gives a result with throughput of $40 \%$.ETAM gives a result with throughput of $30 \%$.

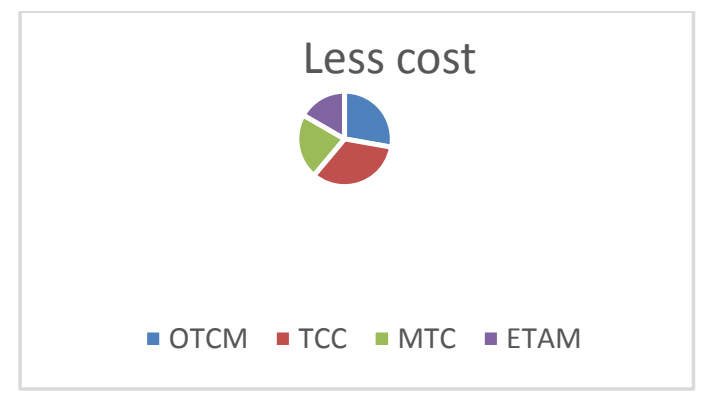

\section{C) Maximum Throughput}

In these experiments maximum throughput. OTCM gives a result with throughput of $40 \%$.TCC gives a result with throughput of 60\%.MTC gives a result with throughput of $55 \%$.ETAM gives a result with throughput of $80 \%$.

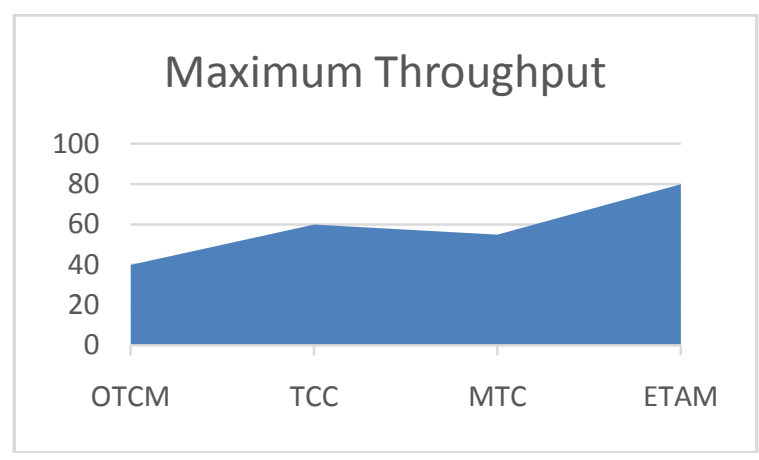

\section{V.CONCLUSION}

The proposed efficient tracking area list management (ETAM) system for $5 \mathrm{G}$ cloud-based mobile networks. The proposed system contains of two parts. fiht part is executed offline. The executed offline is responsible of assigned tracking areas (TAs) to TA lists (TALs). The second one is executed online. The executed online is responsible of the distributed of TALs on user equipment (UEs) during their movements across TAs.For thefirst part, they proposed three keys, which are: (a) F-PAGING favoring the paging overhead over tracking area updated (TAU), (b) F- TAU favoring TAU over paging, and (c) FOTA (i.e., Fair and Optimal Assignment of TALs to TAs) for a solution that uses bargaining game to ensure a fair tradeoff between TAU and paging overhead. For the second part, two solutions are proposed to assigning real time, TALs to different UEs. The computation load is kept lightweight in both solutions not to reduce the network performance. Also, both solutions do not need any additional new messages when assigning TALs to UEs. The first solution taken into account only the priority between TALs. As for the second one, in addition to the priority between TALs, it taken into account the UEs activities (i.e., in terms of incoming communication frequency and mobility patterns) is improved the network performance. The presentation of ETAM is estimated through investigation and imitations, and the achieved results validate its feasibility and ability in achieving its thesis goals, improved the network performance by minimizing the cost related with paging and TAU. 


\section{FUTURE WORK}

The future work persists, they plan to improve the network performance, feasibility and security.

\section{REFERENCES}

[1] MiloudBagaak, AdlenKsentini, “An efficient D2Dbased strategies for Machine Type Communications in 5G mobile systems”, 2016.

[2] Tarik Taleb, Marius Corici, Carlos Parada ,"EASE: EPC as a Service to Ease Mobile Core Network Deployment over Cloud”, 2015.

[3] H.-W. Kang, H.-G. Kang, "Optimization of TAC Configuration in Mobile Communication Systems: A Tabu Search Approach”, 2014.

[4] Tarik Taleb, “Toward Carrier Cloud: Potential, Challenges, and Solutions”, 2014.
[5] Yangming Zhao, Sheng Wang, ShizhongXu, "Load Balance Vs Energy Efficiency in Traffic Engineering: A Game Theoretical Perspective”, 2013.

[6] Tarik Taleb and Andreas Kunz, "Machine Type Communications in 3GPP Networks: Potential, Challenges, and Solutions”, 2012.

[7] Sara ModarresRazavia, Di Yuana ,"Mitigating Signaling Congestion in LTE Location Management by Overlapping Tracking Area Lists”, 2012.

[8] MohsinNawaz, "Exploiting Tracking Area List Concept in Long Term Evolution Networks”, 2010.

[9] Sara ModarresRazavi, Di Yuan, “Dynamic Tracking Area List Configuration and performance Evaluation in LTE”, 2010.

[10] Yan Zhang,Masayuki Fujise, "Location Management Congestion Problem in Wireless Networks”, 2007. 